\title{
PERFIL SENSORIAL DE AGUARDENTE DE CANA EM FUNÇÃO DA DILUIÇÃO E VARIAÇÃO DA ACIDEZ DA AMOSTRA
}

\author{
ELISANGELA MARQUES JERONIMO * \\ HELENA MARIA ANDRÉ BOLINI CARDELLO ** \\ GIL EDUARDO SERRA ***
}

\begin{abstract}
Estudou-se a influência da concentração alcoólica e da acidez no perfil de percepção sensorial de aguardente de cana, visando detectar igualdades ou diferenças na avaliação de atributos típicos de aroma e sabor do produto. Utilizou-se aguardente recémdestilada, que foi submetida a diluição e acidificação com ácido succínico, originando quatro amostras para provas. Concluiu-se que a concentração alcoólica e a acidez não influíram na avaliação sensorial dos atributos típicos da aguardente. A diluição da aguardente não contribuiu para a maximização da percepção de diferenças entre o aroma e o sabor das duas amostras. A variação da acidez não mascarou a expressão sensorial dos atributos devido aos componentes secundários da aguardente, representados principalmente por aldeídos, alcoóis superiores, ésteres e ácidos orgânicos.
\end{abstract}

PALAVRAS-CHAVE: AGUARDENTE-ANÁLISE SENSORIAL; CACHAÇA.

\section{INTRODUÇÃo}

O sabor das bebidas alcoólicas é formado por inúmeros compostos orgânicos voláteis que lhe conferem odor e gosto típico. Esses compostos podem ser divididos em vários grupos de acordo com sua

* Doutoranda do curso de Tecnologia de Alimentos, Departamento de Tecnologia de Alimentos da Faculdade de Engenharia de Alimentos (FEA), Universidade de Campinas (UNICAMP), Campinas, SP (email: elijeronimo@ig.com.br).

** Professora Dra. em Análise Sensorial, do Departamento de Planejamento Alimentar, FEA, UNICAMP, Campinas, SP.

*** Professor Dr. (Aposentado) do Departamento de Tecnologia de Alimentos, Área de Açúcar e Álcool, FEA, UNICAMP, Campinas, SP (gilserra@globo.com). 
natureza química. Alcóois superiores, ácidos graxos e ésteres formam quantitativa e qualitativamente os grupos mais importantes presentes nas bebidas alcoólicas, sendo os alcóois superiores os mais abundantes (LEHTONEN e JOUNELA-ERIKSSON, 1983; BERRY, 1995).

Em aguardentes produzidas por diferentes linhagens de leveduras, OLIVEIRA (2001) verificou variações nos teores e nas relações entre os principais compostos voláteis. Entretanto, tais variações não resultaram em diferenças perceptíveis em relação ao aroma, sabor e impressão global das aguardentes. O sabor perceptível é aquele resultante da combinação de vários componentes. Apesar das bebidas destiladas serem diferenciadas uma das outras por suas características sensoriais não se verifica grande distinção em sua composição química (COLE e NOBLE, 1995).

CARDELLO e FARIA (1998) destacaram que apesar da importância econômica e social da aguardente brasileira são ainda muito escassos os estudos sobre sua qualidade sensorial. Porém, as crescentes exigências do mercado têm estimulado maiores cuidados com a qualidade dessa bebida.

Estudos sobre alimentos e bebidas, visando relacionar os componentes responsáveis pelo aroma e sabor com sua qualidade, são monitorados pela análise sensorial. Essa, continua sendo a única forma de avaliar a aceitação dos mesmos pela percepção humana (STONE e SIDEL, 1998).

Os testes sensoriais são incluídos como garantia de qualidade na indústria de alimentos e bebidas por representarem medida multidimensional integrada. Suas vantagens são: identificar a presença ou ausência de diferenças perceptíveis, definir características sensoriais importantes do produto de forma rápida, detectar particularidades não verificadas por outros procedimentos analíticos e, ainda, ser capaz de avaliar a aceitação de produtos (MUÑOZ et al., 1992).

A análise descritiva quantitativa tem sido bastante empregada para caracterização dos atributos sensoriais de alimentos e bebidas. Foi utilizada por CARDELLO e FARIA (1998) para estudar o perfil sensorial de aguardente de cana envelhecida em tonéis de carvalho. MARCELLINI 
(2000) também utilizou tal metodologia para traçar o perfil sensorial de amostras de aguardentes comerciais, envelhecidas e tradicionais.

Considerando que grupos de fabricantes de aguardente costumam diluir o produto para realização da análise sensorial, com o intuito de salientar características sensoriais negativas, pretendeu-se verificar se essa prática pode ser útil às técnicas tradicionais. Investigou-se a influência da concentração alcoólica e da acidez no perfil sensorial de aguardente, visando maximizar a detecção de igualdades ou diferenças na avaliação (qualitativa e quantitativamente) dos atributos aroma e sabor.

\section{MATERIAL E MÉTODOS}

\subsection{MATERIAL}

A aguardente utilizada foi produzida por OLIVEIRA (2001), na Planta Piloto de Processamento do Departamento de Tecnologia de Alimentos, Universidade de Campinas (UNICAMP). Essa foi elaborada com uma linhagem de Saccharomyces cerevisiae, isolada de produtor artesanal e destilada em alambique de cobre. Escolheu-se a aguardente com base nos padrões de qualidade e identidade da bebida (BRASIL, 1997). Foram considerados baixa acidez, teores adequados de alcoóis superiores em relação ao somatório de componentes secundários e acidez total, além do teor alcoólico padronizado (Tabela 1).

\section{TABELA 1 - TEOR ALCOÓLICO (\%v/v), COMPOSTOS VOLÁTEIS (mg/100 $\mathrm{mL}$ ÁLCOOL ANIDRO) E pH DA AGUARDENTE PRODUZIDA POR OLIVEIRA (2001)}

\begin{tabular}{lllllllll}
\hline Aguardente & "GL $^{1}$ & AcVol $^{2}$ & Actald $^{3}$ & AcEtil $^{4}$ & Prop $^{5}$ & AlSup $^{6}$ & CVT $^{7}$ & pH \\
\hline Sc3 & 39,2 & 38,52 & 14,39 & 14,53 & 11,36 & 316,63 & 384,07 & 4,67 \\
\hline
\end{tabular}

\footnotetext{
${ }^{1}$ Grau Alcoólico (\%v/v); ${ }^{2}$ Acidez volátil em ácido acético; ${ }^{3}$ Acetaldeído; ${ }^{4}$ Acetato de Etila; ${ }^{5}$ Propanol; ${ }^{6}$ Álcoóis superiores; ${ }^{7}$ Compostos Voláteis Totais.

Fonte: OLIVEIRA, 2001.
} 
À partir da aguardente inicial foram preparadas quatro amostras para o estudo sensorial (Tabela 2), as quais diferiram quanto à concentração alcoólica (diluição com água desmineralizada) e acidez (adição de ácido succínico).

\section{TABELA 2 - AMOSTRAS DE AGUARDENTE PREPARADAS PARA O ESTUDO SENSORIAL, VARIANDO O TEOR ALCOÓLICO E A ACIDEZ}

\begin{tabular}{lcccc}
\hline Amostras & GN/SA & GD/SA & GN/CA & GD/CA ${ }^{(1)}$ \\
\% etanol v/v, a $20^{\circ} \mathrm{C}$ & 39 & 20 & 39 & 20 \\
mg / Æido acØico/100 mL etanol & 48 & 24 & 90 & 48 \\
mg / Æido acØico/100 mL aguardente & 18,5 & 10 & 35 & 19 \\
\hline
\end{tabular}

(1) GN/SA = Grau Alcoólico Normal/Sem Acidificação; GD/SA = Grau Alcoólico Diluído/Sem Acidificação; GN/CA = Grau Alcoólico Normal/Com Acidificação; GD/ $\mathrm{CA}=$ Grau Alcoólico Diluído/Com Acidificação;

\subsection{MÉTODOS}

\subsubsection{Análise Descritiva Quantitativa}

\subsubsection{Pré-seleção da equipe}

Efetuou-se pré-seleção de 25 candidatos para compor a equipe de julgadores para a análise descritiva quantitativa, mediante oito sessões de testes triangulares. Foram utilizadas duas amostras de aguardentes com diferença significativa entre si ao nível de $0,1 \%$. Os julgadores que obtiveram mais de $70 \%$ de acertos nos oito testes aplicados foram selecionados. Para a análise dos dados do teste triangular foi utilizado o teste $\chi^{2}$ (qui-quadrado), conforme ROESSLER e ALDER (1972). Além desses resultados foram consideradas a disposição e disponibilidade de tempo para participar de treinamento adequado. 


\subsubsection{Levantamento dos atributos}

Doze candidatos efetuaram o levantamento dos termos descritivos pelo método de rede de Kelly, descrito em MOSKOWITZ (1983). Os julgadores receberam as amostras aos pares (todas as combinações) e listaram as similaridades e as diferenças em relação ao aroma e ao sabor em ficha apropriada.

Depois do levantamento dos termos, a equipe reuniu-se várias vezes em debate aberto para escolha dos termos mais apropriados e importantes que realmente descrevessem os atributos das amostras.

Elaborou-se ficha de avaliação a partir dos atributos escolhidos usando escala não-estruturada de 9 centímetros, ancorada nos pontos extremos pelos termos "fraco" ou "nenhum" e "forte" para cada atributo.

\subsubsection{Definições e referências dos atributos selecionados}

Foram realizadas várias sessões de treinamento para padronização das notas dos 12 julgadores de forma que se situassem na mesma região da escala. Essa padronização é realizada com a apresentação de amostras-referência dos extremos das escalas. Tais amostras foram escolhidas em função dos termos descritivos e sugestões da equipe.

\subsubsection{Seleção dos julgadores}

Os testes para a seleção da equipe definitiva para a análise descritiva quantitativa foram realizados com a ficha contendo as escalas de intensidade para os termos definidos.

Os julgadores foram selecionados com base no poder de discriminação entre as amostras, repetibilidade e concordância com a equipe. Efetuouse análise de variância (ANOVA) das duas fontes de variação (amostra e repetição) para cada atributo e para cada julgador, obtendo os valores para $F_{\text {amostra }}$ e $F_{\text {repetição }}$ de cada julgador. Foram selecionados os provadores com valores de $F_{\text {amostra }}$ significativo $(p \leq 0,50)$ e $F_{\text {repetição }}$ nãosignificativo $(p>0,05)$ e concordância com as médias da equipe. 
As amostras foram servidas a cada julgador (equipe selecionada e treinada) em laboratório com cabines individuais, usando-se cálices incolores de vidro transparente e codificados. Os cálices estavam cobertos com vidro de relógio, os quais foram retirados no momento do teste. Todas as amostras foram apresentadas de forma monádica, com três repetições aleatorizadas, num total de 12 sessões por julgador.

\subsubsection{Análise dos dados}

Os dados coletados foram submetidos a análise de variância (ANOVA) de dois fatores (amostra e julgador) com interação para cada atributo. Foi aplicado o teste de Tukey para comparação das médias das amostras e efetuada a Análise de Componentes Principais. As análises estatísticas foram realizadas mediante programas do pacote estatístico SAS (SAS, 1993).

\section{RESULTADOS E DISCUSSÃO}

A partir da lista obtida pelo método de rede, os atributos escolhidos pelos membros da equipe sensorial (pré-selecionada) foram: aroma alcoólico, aroma agressivo, sabor alcoólico, sabor agressivo, sabor adocicado, gosto amargo e gosto ácido (Tabela 3). Com os termos descritores gerados foi elaborada a ficha de avaliação para traçar o perfil sensorial das amostras.

Os julgadores participaram de várias sessões de treinamento para os testes. Em cada uma tomavam contato com as referências e amostras para relembrar os pontos extremos da escala para cada termo descritor.

\subsection{SELEÇÃO DOS JULGADORES PARA A ANÁLISE DESCRITIVA QUANTITATIVA}

Na Tabela 4 estão expressos os resultados dos níveis de significância (p) de $F_{\text {amostra }}$ para cada julgador em relação a cada atributo. Foi 
observada variação no poder de discriminação entre os provadores, porém 11 dos 12 julgadores apresentaram valores de $F_{\text {amostra }}$ significativos ao nível de $50 \%$ em pelo menos seis dos sete atributos avaliados. Assim, demonstraram capacidade para discriminar as amostras a esse nível de significância em $86 \%$ dos parâmetros, o que representa nível de discriminação aceitável (Tabela 4). De acordo com esses critérios todos os 12 candidatos seriam selecionados, mas onze julgadores compuseram a equipe sensorial definitivamente.

\section{TABELA 3 - DEFINIÇÕES E REFERÊNCIAS PARA OS TERMOS} DESCRITORES LEVANTADOS PELOS JULGADORES

\begin{tabular}{|c|c|c|}
\hline Termo Descritor & Defini ${ }^{a} 0$ & ReferC̄acias \\
\hline $\begin{array}{l}\text { Aroma Alco lico } \\
\text { (AAL) }\end{array}$ & Aroma caracter stico de etanol & $\begin{array}{l}\text { "Fraco" = etanol } 20 \% \\
\text { "Forte" = etanol } 45 \%\end{array}$ \\
\hline $\begin{array}{l}\text { Aroma Agressivo } \\
\text { (AAG) }\end{array}$ & Impacto agressivo do aroma inicial & $\begin{array}{l}\text { "Fraco" = aguardente } \\
\text { envelhecida } 20 \% \\
\text { "Forte" = etanol } 45 \%\end{array}$ \\
\hline $\begin{array}{l}\text { Sabor Alco lico } \\
\text { (SAL) }\end{array}$ & $\begin{array}{l}\text { Sabor caracter stico de solu I es } \\
\text { alco licas }\end{array}$ & $\begin{array}{l}\text { "Fraco" = etanol 18\% } \\
\text { "Forte" = etanol } 44 \%\end{array}$ \\
\hline $\begin{array}{l}\text { Sabor Agressivo } \\
\text { (SAG) }\end{array}$ & Impacto agressivo do sabor inicial & $\begin{array}{l}\text { "Fraco" = aguardente } \\
\text { envelhecida } 18 \% \\
\text { "Forte" = etanol } 45 \%\end{array}$ \\
\hline $\begin{array}{l}\text { Sabor Adocicado } \\
\text { (SAD) }\end{array}$ & $\begin{array}{l}\text { Gosto doce percebido no instante } \\
\text { em que determinada subst ncia } \\
\text { entra em contato com os botı es } \\
\text { gustativos }\end{array}$ & $\begin{array}{l}\text { "Fraco" = etanol } 20 \% \\
\text { "Forte" = etanol } 20 \% \\
\text { contendo } 4 \% \text { de agente } \\
\text { ado ante }\end{array}$ \\
\hline $\begin{array}{l}\text { Gosto } \\
\text { Amargo } \\
\text { (GAM) }\end{array}$ & $\begin{array}{l}\text { Gosto amargo, caracter stico de } \\
\text { cafe na }\end{array}$ & $\begin{array}{l}\text { "Nenhum" = Agua destilada } \\
\text { "Forte" = etanol } 38 \% \text { com } \\
\text { cafe na a } 0,5 \%\end{array}$ \\
\hline $\begin{array}{l}\text { Gosto } \\
\text { cido } \\
(\mathrm{GAC})\end{array}$ & $\begin{array}{l}\text { Gosto Aeido, caracter stico de } \\
\text { frutas c tricas }\end{array}$ & $\begin{array}{l}\text { "Fraco" = etanol } 20 \% \\
\text { "Forte" = etanol } 20 \% \\
\text { contendo } 0,15 \% \text { de Aeido } \\
\text { c trico }\end{array}$ \\
\hline
\end{tabular}

Na Tabela 5 estão expressos os resultados dos níveis de significância (p) de $F_{\text {repeticão }}$ para cada julgador, em relação a cada atributo. Foram selecionados os julgadores com os valores de $p$ de $F_{\text {repetição }}$ não significativo $(p>0,05)$. Julgadores com $p$ de $F_{\text {repetição }} \leq 0,05$ não devem 
ser selecionados devido ao baixo nível de repetibilidade. De maneira geral, os resultados revelaram alta reprodutibilidade, pois apenas um dos julgadores apresentou valor de $\mathrm{F}_{\text {repetição }}$ significativo $(\mathrm{p} \leq 0,05)$ para mais de um atributo.

\section{TABELA 4 - RESULTADOS DOS NÍVEIS DE SIGNIFICÂNCIA (P) DE F AMOSTRA PARA CADA JULGADOR/ATRIBUTO}

\begin{tabular}{cccccccc}
\hline Julgador & AAL & AAG & SAL & SAG & SAD & GAM & GAC $^{(1)}$ \\
\hline P1 & 0,0006 & 0,0494 & 0,0194 & 0,1282 & 0,0002 & 0,7171 & 0,0114 \\
P2 & $<, 0001$ & $<, 0001$ & $<, 0001$ & $<, 0001$ & $<, 0001$ & 0,0265 & 0,0186 \\
P3 & 0,0057 & 0,0074 & $<, 0001$ & 0,0002 & 0,0187 & 0,2146 & 0,1783 \\
P4 & 0,0068 & 0,0190 & $<, 0001$ & 0,0002 & 0,0056 & 0,0170 & 0,3581 \\
P5 & 0,0001 & $<, 0001$ & $<, 0001$ & $<, 0001$ & 0,9015 & 0,5259 & 0,0284 \\
P6 & 0,0037 & 0,0195 & $<, 0001$ & 0,0025 & 0,1858 & 0,4065 & 0,0014 \\
P7 & 0,0057 & 0,0004 & $<, 0001$ & $<, 0001$ & 0,0515 & 0,2586 & 0,1441 \\
P8 & 0,1046 & 0,0018 & 0,0153 & 0,0243 & 0,1040 & $<, 0001$ & 0,0081 \\
P9 & 0,0391 & 0,0002 & $<, 0001$ & $<, 0001$ & 0,0640 & 0,0139 & 0,0238 \\
P10 & 0,0001 & $<, 0001$ & 0,0012 & $<, 0001$ & 0,1523 & 0,0017 & 0,3942 \\
P11 & $<, 0001$ & $<, 0001$ & 0,0028 & $<, 0001$ & 0,1184 & $<, 0001$ & 0,0005 \\
P12 & 0,0003 & 0,0102 & $<, 0001$ & 0,0059 & 0,1714 & 0,0855 & 0,5538 \\
\hline
\end{tabular}

$\mathrm{AAL}=$ Aroma Alcoólico; $\mathrm{AGG}=$ Aroma Agressivo; $\mathrm{AS}=$ Sabor Alcoólico; $\mathrm{SAG}=$ Sabor Agressivo; $\mathrm{SAD}=$ Sabor Adocicado; $\mathrm{GAM}=$ Gosto Amargo; $\mathrm{GAC}=$ Gosto Ácido.

\section{TABELA 5 - RESULTADOS DOS NÍVEIS DE SIGNIFICÂNCIA (P) DE F REPETIÇÃO PARA CADA JULGADOR/ATRIBUTO}

\begin{tabular}{clllllll}
\hline Julgador & AAL & AAG & SAL & SAG & SAD & GAM & GAC \\
\hline P1 & 0,0394 & 0,5120 & 0,6439 & 0,9947 & 0,1774 & 0,8801 & 0,4637 \\
P2 & 0,9929 & 0,3904 & 0,0360 & 1,0000 & 0,0085 & 0,9489 & 0,3585 \\
P3 & 0,0183 & 0,0070 & 0,0415 & 0,1535 & 0,2103 & 0,6420 & 0,4363 \\
P4 & 0,7965 & 0,5936 & 0,9884 & 0,9512 & 0,1717 & 0,7108 & 0,5753 \\
P5 & 0,5344 & 0,0298 & 0,6975 & 0,0889 & 0,9998 & 0,6054 & 0,3497 \\
P6 & 0,3421 & 0,2174 & 0,5846 & 0,4287 & 0,2874 & 0,0974 & 0,2144 \\
P7 & 0,5827 & 0,1512 & 0,8237 & 0,3423 & 0,6499 & 0,9880 & 0,4435 \\
P8 & 0,9997 & 0,9370 & 0,7825 & 0,9600 & 0,2194 & 0,9677 & 0,8017 \\
P9 & 0,9981 & 0,5532 & 0,3732 & 0,2424 & 0,9996 & 0,7297 & 0,5181 \\
P10 & 0,1275 & 0,7301 & 0,5041 & 0,9090 & 0,3161 & 0,2102 & 0,0214 \\
P11 & 0,1503 & 0,2594 & 0,2109 & 0,0032 & 0,9152 & 0,9535 & 0,0529 \\
P12 & 0,7012 & 0,2617 & 0,1600 & 0,2390 & 0,0960 & 0,0096 & 0,3661 \\
\hline
\end{tabular}

$\mathrm{AAL}=$ Aroma Alcoólico; $\mathrm{AGG}=$ Aroma Agressivo; $\mathrm{AS}=$ Sabor Alcoólico; $\mathrm{SAG}=$ Sabor Agressivo; $\mathrm{SAD}$ = Sabor Adocicado; $\mathrm{GAM}=$ Gosto Amargo; $\mathrm{GAC}=$ Gosto Ácido. 


\subsection{ANÁLISE DESCRITIVA QUANTITATIVA}

A Tabela 6 contém os resultados das médias dos atributos para as amostras avaliadas.

\section{TABELA 6 - MÉDIA DOS ATRIBUTOS PARA AS AGUARDENTES E COMPARAÇÃO PELO TESTE DE TUKEY PARA AGUARDENTES COM GRADUAÇÃO NORMAL E DILUÍDA, SEM OU COM ACIDIFICAÇÃO}

\begin{tabular}{llllllll}
\hline Amostra & AAL & AAG & SAL & SAG & SAD & GAM & GAC \\
\hline GN/SA & $7,17 \mathrm{a}$ & $7,22 \mathrm{a}$ & $7,37 \mathrm{a}$ & $7,50 \mathrm{a}$ & $2,73 \mathrm{a}$ & $3,75 \mathrm{~b}$ & $3,94 \mathrm{bc}$ \\
GD/SA & $1,73 \mathrm{~b}$ & $1,37 \mathrm{~b}$ & $1,86 \mathrm{~b}$ & $1,42 \mathrm{~b}$ & $2,85 \mathrm{a}$ & $2,56 \mathrm{c}$ & $3,21 \mathrm{c}$ \\
GN/CA & $7,38 \mathrm{a}$ & $7,32 \mathrm{a}$ & $7,34 \mathrm{a}$ & $7,67 \mathrm{a}$ & $2,66 \mathrm{a}$ & $4,71 \mathrm{a}$ & $5,05 \mathrm{a}$ \\
GD/CA & $2,06 \mathrm{~b}$ & $1,61 \mathrm{~b}$ & $2,43 \mathrm{~b}$ & $2,42 \mathrm{~b}$ & $3,33 \mathrm{a}$ & $3,63 \mathrm{~b}$ & $4,25 \mathrm{ab}$ \\
\hline \multirow{2}{*}{ DMS } & 0,5258 & 0,5925 & 0,5980 & 0,5214 & 0,9058 & 1,0619 & 0,8731 \\
& & & & & & & \\
\hline
\end{tabular}

Médias seguidas pela mesma letra na mesma coluna não diferem entre si estatisticamente $(p \leq 0,05)$; DMS = diferença mínima significativa entre as médias. $\mathrm{AAL}=$ Aroma Alcoólico; $\mathrm{AGG}$ = Aroma Agressivo; $\mathrm{AS}=$ Sabor Alcoólico; $\mathrm{SAG}=$ Sabor Agressivo; SAD = Sabor Adocicado; GAM = Gosto Amargo; GAC = Gosto Ácido.

A análise de variância dos resultados (fontes de variação: julgador, amostra e interação entre amostra e julgador) revelou diferença significativa entre as amostras 1 e 3 (Aguardentes a 39\% de etanol) e as amostras 2 e 4 (Aguardentes a $20 \%$ de etanol) em relação ao aroma alcoólico (AAL), aroma agressivo (AAG), sabor alcoólico (SAL) e sabor agressivo (SAG).

Para o atributo gosto ácido (GAC), a amostra 3 (Aguardente Acidificada a $39 \%$ de etanol) obteve média significativamente maior $(p \leq 0,05)$ em relação as amostras não-acidificadas (Aguardentes a 39\% e $20 \%$ de etanol, 1 e 2, respectivamente). A amostra 4 (Aguardente Acidificada a $20 \%$ de etanol) também obteve média significativamente maior em relação à amostra 2 (Aguardente a 20\% etanol).

Não houve diferença significativa entre as quatro amostras em relação ao atributo sabor adocicado (SAD). 
A amostra 3 (Aguardente Acidificada a 39\% de etanol) obteve média significativamente maior $(p \leq 0,05)$ para o atributo gosto amargo (GAM) em relação à amostra 4 (Aguardente Acidificada a 20\% de etanol) e às amostras não-acidificadas (1 e 3).

A Figura 1 evidencia que as aguardentes com $39^{\circ} \mathrm{GL}$ (amostras 1 e 3 ) apresentaram aroma alcoólico, aroma agressivo, sabor alcoólico e sabor agressivo de forma mais pronunciada que as demais amostras.

FIGURA 1 - PERFIL SENSORIAL EM GRÁFICO ESTRELA COM AS MÉDIAS DOS ATRIBUTOS DAS AGUARDENTES COM DIFERENTES GRADUAÇÕES ALCOÓLICAS E ACIDEZ

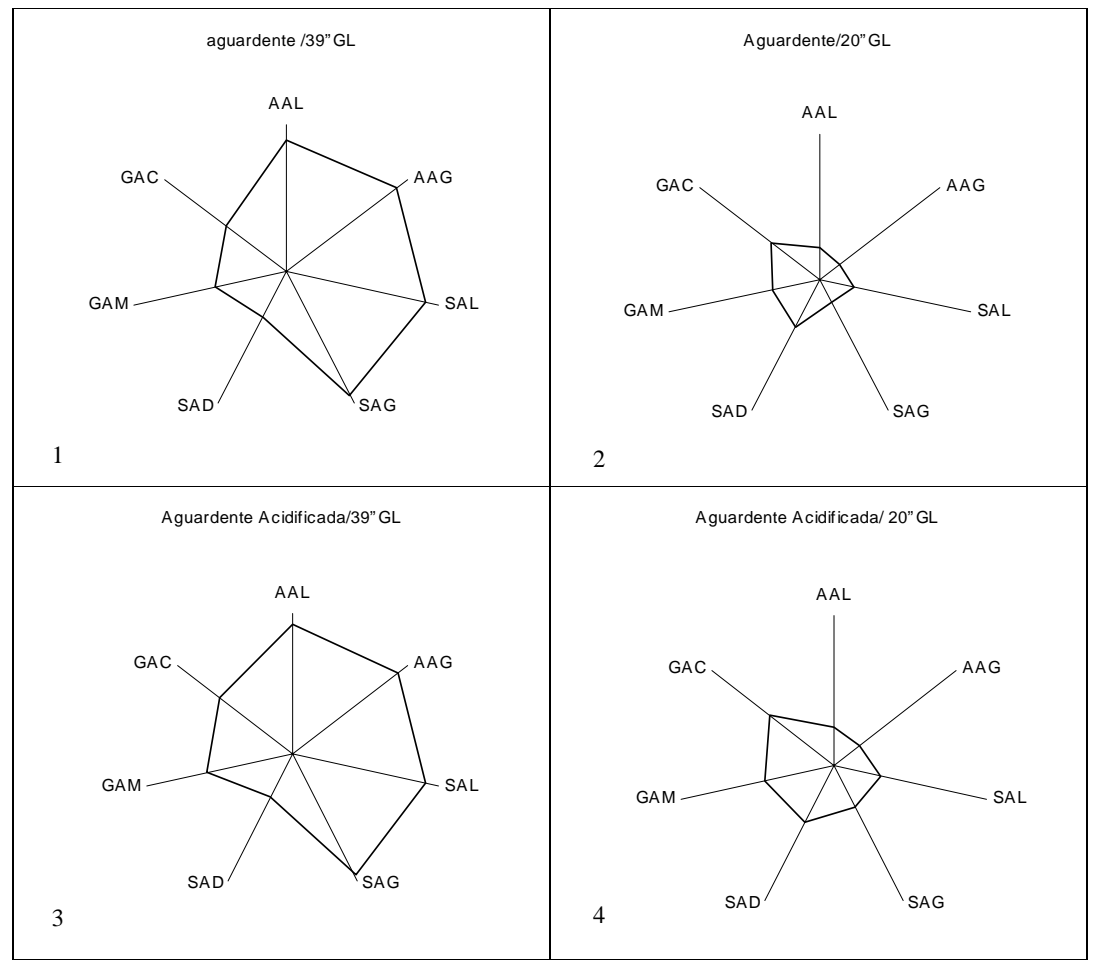

1 = aguardente $39^{\circ} \mathrm{GL} ; 2$ = aguardente $20^{\circ} \mathrm{GL} ; 3$ = aguardente acidificada $39^{\circ} \mathrm{GL}$; 4 = aguardente acidificada 20ㅡ․; $A A L=$ Aroma Alcoólico; $A G G$ = Aroma Agressivo; $\mathrm{AS}$ = Sabor Alcoólico; SAG = Sabor Agressivo; SAD = Sabor Adocicado; GAM = Gosto Amargo; GAC = Gosto Ácido. 
Todas as aguardentes demonstraram praticamente a mesma intensidade para os atributos sabor adocicado. O gosto ácido foi percebido com a mesma intensidade nas aguardentes acidificadas a $39 \%$ e a $20 \%$ de etanol (amostras 3 e 4 ).

A representação gráfica da Análise de Componentes Principais (ACP) mostra as configurações dos atributos sensoriais das aguardentes e sua distribuição bidimensional (Figura 2).

\section{FIGURA 2 - GRÁFICO BIDIMENSIONAL DA ANÁLISE DE COMPONENTES PRINCIPAIS DAS AGUARDENTES}

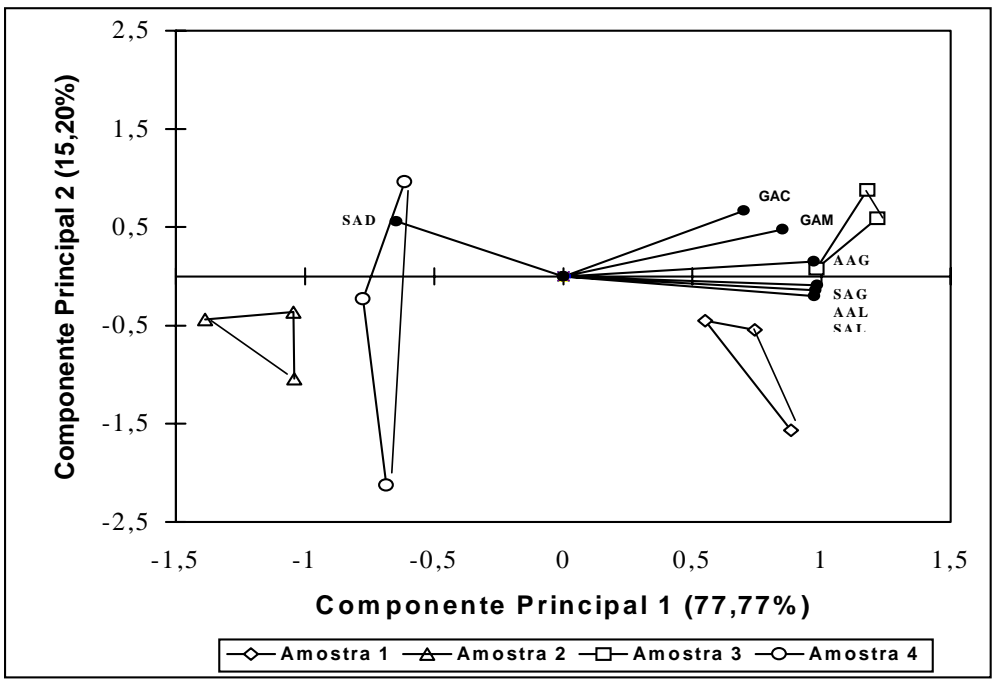

1 = aguardente $39^{\circ} \mathrm{GL} ; 2$ = aguardente $20^{\circ} \mathrm{GL} ; 3$ = aguardente acidificada $39^{\circ} \mathrm{GL}$; 4 = aguardente acidificada $20^{\circ} \mathrm{GL} ; \mathrm{AAL}=$ Aroma Alcoólico; $\mathrm{AGG}=$ Aroma Agressivo; AS = Sabor Alcoólico; SAG = Sabor Agressivo; SAD = Sabor Adocicado; $\mathrm{GAM}=$ Gosto Amargo; GAC = Gosto Ácido.

Verificou-se que $77,77 \%$ da variação ocorrida entre as amostras foi explicada pelo primeiro eixo (Componente Principal 1). Os Componentes Principais 1 e 2 explicaram juntos 92,97\% da variação entre as amostras, evidenciando que os descritores empregados discriminaram satisfatoriamente as amostras analisadas. 
$\mathrm{Na} \mathrm{ACP}$, vetores com tamanho reduzido indicam atributos nas quais as amostras pouco diferem entre si e portanto apresentam importância semelhante para explicar as variações entre as amostras (Figura 2).

De acordo com MUÑOZ et al. (1992), vetores com medidas mais distantes de zero correspondem à variações com maior influência sobre o valor do Componente Principal. Já vetores mais próximos de zero indicam variável com pequena influência sobre o Componente Principal. Portanto, nem todos os atributos gerados para as amostras de aguardentes corresponderam à variações com grande influência.

Os atributos GAC, GAM, SAG, SAM, AAG, AAL (positivamente) e SAD (negativamente) contribuíram com maior peso sobre a variabilidade associada ao primeiro eixo.

As amostras 1 e 3 mostraram-se bem distintas das amostras 2 e 4, marcadas pelas localizações bem definidas de cada uma no gráfico bidimensional.

O experimento apresentou repetibilidade razoável, evidenciada pelo agrupamento dos pontos representativos de cada amostra.

$\mathrm{Na}$ ACP, as amostras localizaram-se próximas dos vetores (atributos) que as caracterizam. Portanto, as amostras 1 e 3 caracterizaram-se por SAG, SAL, AAL, AAG, GAM e GAC, a amostra 3 por GAM, GAC e SAD e a amostra 2 apenas pelo SAD.

Os resultados obtidos mostraram que a concentração alcoólica e a acidificação das aguardentes não resultaram na atribuição de qualidade diferenciada para os produtos.

\section{CONCLUSÃO}

A concentração alcoólica e a acidez não influíram na avaliação sensorial dos atributos típicos da aguardente. A diluição da aguardente não contribuiu para a maximização da percepção de diferenças entre as amostras. A variação da acidez também não mascarou a expressão sensorial dos atributos devido aos componentes secundários da aguardente representados principalmente por aldeídos, alcoóis superiores, ésteres e ácidos orgânicos. 


\begin{abstract}
SENSORY PROFILE OF SUGARCANE SPIRIT IN FUNCTION TO SAMPLE DILUTION AND ACIDITY VARIATION

The influence of alcoholic concentration and acidity on the sensory perception profile of sugarcane spirit was studied, aiming to detect similarities and differences in the evaluation of product flavor and aroma typical attributes. Recent prepared sugarcane spirit was utilized, which was submitted to dilution and acidification with succinic acid, originating four samples for tests. It was concluded that the alcoholic concentration and acidity didn't influence the sensory evaluation of the typical attributes of the spirit. The spirit dilution didn't contribute for the maximization of differences perception among aroma and flavor of two samples. The acidity variation didn't disguise the attributes sensory expression due to the secondary components of the spirit, represented mainly by aldehydes, superior alcohols, esters and organic acids.
\end{abstract}

KEY-WORDS: SPIRIT-SENSORY ANALYSIS; SUGARCANE SPIRIT.

\title{
REFERÊNCIAS
}

1 BERRY, D.R. Alcoholic beverage fermentations. In: LEA, A.G.H.; PIGGOTT, J.R. Fermented Beverage Production. London: Blackie Academic \& Professional, 1995. p. 32-44.

2 BRASIL. Decreto n.2314, de 04 de setembro de 1997, do Ministério da Agricultura. Dispõe sobre o registro, classificação, padronização, produção e fiscalização das bebidas. Diário Oficial [da] República Federativa do Brasil, Brasilia, 5 de setembro de 1997.

3 CARDELLO, H. M.A. B.; FARIA, J.B. Análise descritiva quantitativa da aguardente de cana durante o envelhecimento em tonel de carvalho (Quercus alba L.). Ciên. Tec. Alim., v.18, n.2, p.169-175, maio/jul., 1998.

4 COLE, V. C.; NOBLE, A. C. Flavor chemistry and assessment. In: LEA, A. G. H.; PIGGOTT, J. R. Fermented beverage production. London: Blackie Academic \& Professional, 1995. p. 361-385.

5 LEHTONEN, M.; JOUNELA-ERIKSSON, P. Volatile and non-volatile compounds in the flavour of alcoholic beverages. In: PIGGOTT, J.R. Flavour of distilled beverages: origin and development. Flórida: Verlag Chemie, 1983. p. 64-78.

6 MARCELLINI, P.S. Análise descritiva quantitativa de aguardentes de cana (Saccharum spp) comerciais e destiladas em alambiques de cobre e aço-inoxidável. Araraquara, 2000. 77 p. Dissertação (Mestrado) Faculdade de Ciências Farmacêuticas, UNESP.

7 MOSKOWITZ, H. R. Product testing and sensory evaluation of foods: 
marketing and R \& D approaches. Westport: Food \& Nutrition Press, 1983. $605 \mathrm{p}$.

8 MUÑOZ, A. M.; CIVILLE, G. V., CARR, B. T. Sensory evaluation in quality control. New York: Van Nostrand Reinhold, 1992. 240 p.

9 OLIVEIRA, E. S. Características fermentativas, formação de compostos voláteis e qualidade da aguardente de cana obtida por linhagens de leveduras isoladas em destilarias artesanais. Campinas, 2001. 135 p. Tese (Doutorado em Tecnologia de Alimentos) - Faculdade de Engenharia de Alimentos, UNICAMP.

10 ROESSLER, E. B.; ALDER, H. L. Introduction to probabiity and statistics. $5^{\text {th }}$ ed. San Francisco: W. C. Freeman, 1972. p. 195-220.

11 SAS Institute SAS user's guide: statistics. Cary, USA, 1993.

12 STONE, H.; SIDEL, J. L. Sensory evaluation pratices. $2^{\text {nd }}$ ed. San Diego: Academic Press, 1993. $338 \mathrm{p}$.

\section{AGRADECIMENTO}

Agradecemos à Fundação de Amparo à Pesquisa do Estado de São Paulo (FAPESP) pelo suporte financeiro e pela bolsa de doutoramento concedida ao primeiro autor. 